\title{
Correction to: Pattern Recognition and Machine Intelligence
}

\author{
Bhabesh Deka (D), Pradipta Maji (iD, Sushmita Mitra (D), \\ Dhruba Kumar Bhattacharyya (D), Prabin Kumar Bora, \\ and Sankar Kumar Pal (D)
}

\section{Correction to: \\ B. Deka et al. (Eds.): Pattern Recognition and Machine Intelligence, LNCS 11942, https://doi.org/10.1007/978-3-030-34872-4}

In Chapter "QIBDS Net: A Quantum-Inspired Bi-Directional Self-supervised Neural Network Architecture for Automatic Brain MR Image Segmentation":

The original version of this chapter was revised. Titles that were originally not visible in the reference section are now displayed properly.

In Chapter "RBM Based Joke Recommendation System and Joke Reader Segmentation":

The original version of this chapter was revised. Figure 6 of this chapter was incorrect and it has been replaced. Few changes have been made in the text part of Section 3.

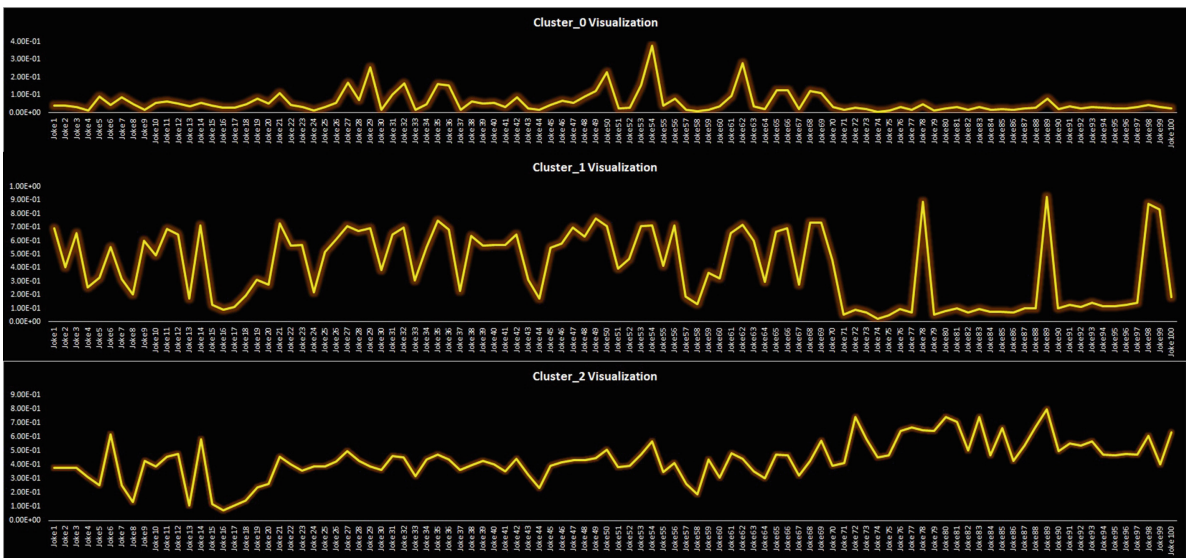

Fig. 6. Joke-reader segment visualization

The updated version of these chapters can be found at https://doi.org/10.1007/978-3-030-34872-4_10 https://doi.org/10.1007/978-3-030-34872-4_26 